\title{
The relationship between dietary micro and macronutrients intake and cell proliferation in the colon
}

\author{
C. McGuinness, D. O’Shea, A. R. Gunawardene, E. A. Williams and B. M. Corfe \\ Human Nutrition Unit, Department of Oncology, University of Sheffield, Sheffield, S10 2RX, UK
}

Colorectal cancer, a leading cause of death worldwide, displays increased incidence in individuals adopting a western style diet ${ }^{(1)}$. Diet is believed to be a major factor in the aetiology of colorectal cancer, with a number of nutrients implicated as causative and preventative factors of colorectal cancer ${ }^{(2)}$. One key cellular process identified as dysregulated in the process of colorectal carcinogenesis is cell proliferation and division. As part of a larger study to unpack the axis from fibre to cancer ${ }^{(3)}$, we recruited fifty-six participants with a normal large bowel pathology from gastroenterology outpatient clinics and collected Carnoy's-fixed colorectal biopsies for whole mount analysis of mitosis and formalin-fixed biopsies for Ki67 staining, a marker of cycling cells. Habitual dietary intake was assessed using a modified version of the EPIC FFQ. The relationships between estimated intakes of fat, protein, carbohydrate, fibre, riboflavin, folate, Ca and alcohol and colonocyte cell proliferative indices were explored using Pearson's correlation coefficient.

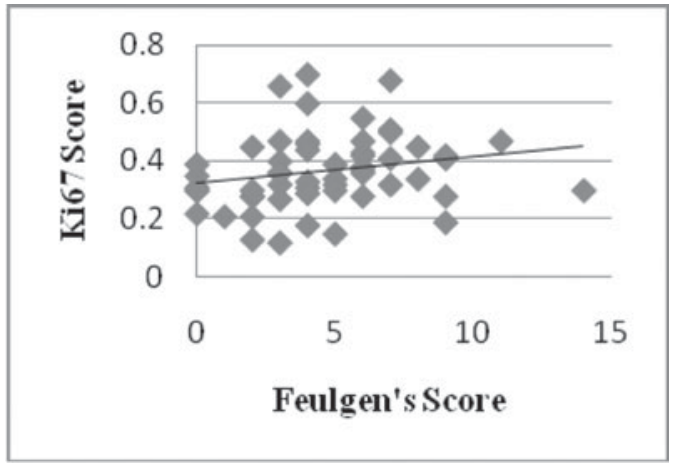

Table 1. Correlation co-efficient between age, BMI, dietary intake of nutrients and markers of colonoctype proliferation

\begin{tabular}{lrr}
\hline & \multicolumn{1}{c}{ Feulgen's r(p) } & \multicolumn{1}{c}{ Ki67 r(p) } \\
\hline Age & $0.176(0.194)$ & $0.239(0.076)$ \\
BMI & $-0.096(0.482)$ & $0.069(0.614)$ \\
Protein & $0.157(0.249)$ & $0.093(0.493)$ \\
Carbohydrates & $0.118(0.386)$ & $0.151(0.267)$ \\
Fat & $0.112(0.369)$ & $0.046(0.737)$ \\
Fibre & $-0.135(0.323)$ & $-0.028(0.836)$ \\
Riboflavin & $0.064(0.640)$ & $0.092(0.498)$ \\
Folate & $0.105(0.442)$ & $0.098(0.475)$ \\
Alcohol & $-0.092(0.502)$ & $-0.099(0.470)$ \\
Calcium & $0.156(0.252)$ & $0.077(0.570)$ \\
\hline
\end{tabular}

Fig. 1. Relationship between proliferative indices in the colon.

For mitosis (Feulgen's positive count) no significant relationships were observed between any nutrient and mitosis. For cell cycle (Ki67-positive count) there was no significant relationship observed between any nutrient and cell cycle, however, age shows a near significant relationship (Table 1). The relationship between the two proliferative indices showed a surprisingly weak positive correlation (Fig. 1).

In conclusion, there is no strong evidence to support a relationship between nutrient intake and markers of cell proliferation in the colon, larger studies may be required to identify such. These data may implicate other mechanisms (such as alterations in the colon microenvironment, stimulation of detoxification and of apoptosis) as mediators of nutritional chemoprevention. The relationship between proliferative indices merits further study.

1. Marchand LL (1999) J Natl Cancer Inst Monogr 26, 101-105.

2. Marmot M, Atinmo T et al. (2007) World Cancer Research Fund/American Institute for Cancer Research. Food, Nutrition, Physical Activity, and the Prevention of Cancer; a Global Perspective. Washington DC: AICR.

3. Corfe BM, Williams EA, Bury JP et al. (2009) BMC Cancer 9, 332. 